\title{
1810 A Study on Uncertainty Propagation Modeling in Flow Analysis 流れ解析における不確かさ伝播モデルの検証
}

\author{
Hiromasa Kato \\ Department of Mechanical Engineering, Iwate University, Morioka, Japan, \\ Rajan Filomeno Coelho \\ BATir Department, Université Libre de Bruxelles, Brussels, Belgium.
}

\section{Introduction}

In order to achieve higher performance in fluid machines, simulation-based aerodynamic shape optimization is now a commonplace in industry, taking advantage of the rapid progress in computational capacity and efficient numerical optimization algorithms. Computational fluid dynamics (CFD) codes are used for the evaluation of objective functions as well as constraints. One common pitfall of such simulation-based optimization is overoptimization, a situation where an optimized shape only performs well at prespecified operating conditions. [1] Thus it has now been commonly recognized that numerical optimization must incorporate a notion of robustness in the evalution of objective and constraint functions by not only optimizing the mean but also by controlling the variance of the objective functions. On the other hand, CFD codes are notorious for their heavy computational requirements, often taking hours or days for an evaluation of a single case, the fact which puts a severe constraint on the choice of numerical methods that can be used to estimate statistical measures such as mean and variance.

One promising approach recently attracting attention is the use of Polynomial Chaos Expansion (PCE) [2, 3, 4], which allows the estimation of mean and variance at a cost far below that of Monte Carlo simulation and can be used in an non-intrusive manner, i.e. not requiring any modification of underlying simulation codes. The present study aims to reveal real-world performance of PCE-based approaches as applied to the estimation of mean and variance of supersonic impulse turbine stage efficiency when its rotational speed is a random variable, i.e. uncertain.

\section{Polynomial Chaos Expansion}

A stochastic variable, $f^{*}$, which is a function of a deterministic vector, $\mathbf{x}$, and a random variable, $\xi$, can be approximated through a modal decomposition using PCE as follows.

$$
f^{*}(\mathbf{x}, \xi)=\sum_{i=0}^{P} f_{i}(\mathbf{x}) \Psi_{i}(\xi)
$$

where $f_{i}$ are the deterministic coefficients and $\Psi_{i}$ the stochastic basis functions. There are $P+1$ terms present in Eq. 1 and $P$ is a function of the number of random variables, $m$, and the order of the expansion, $n$, as follows.

$$
P+1=\frac{(m+n) !}{m ! n !}
$$

If we assume a normal distribution for the random variable, $\xi$, the basis functions are Hermite polynomials. Though it is not a requirement for $\xi$ to be normally distributed, we only treat such a source of randomness in the discussion that follows. The most important feature of $\mathrm{PCE}$ is that the basis functions are orthogonal with measure $p_{N}(\xi)$ (probability density function of $\xi$ ),

$$
\left\langle\Psi_{i}(\xi), \Psi_{j}(\xi)\right\rangle=\int_{-\infty}^{\infty} \Psi_{i}(\xi) \Psi_{j}(\xi) p_{N}(\xi) d \xi=\delta_{i j}
$$

where we denote the inner product above with $\langle$,$\rangle and$ $\delta_{i j}$ is the Kronecker delta. For reference, Hermite polynomials of a scalar random variable $\xi$ are given as follows.

$\Psi_{0}(\xi)=1, \Psi_{1}(\xi)=\xi, \Psi_{2}(\xi)=1-\xi^{2}, \Psi_{3}(\xi)=\xi^{3}-3 \xi, \ldots$

The corresponding inner products, $\left\langle\Psi_{i}^{2}(\xi)\right\rangle$, are

$$
\left\langle\Psi_{0}^{2}(\xi)\right\rangle=1,\left\langle\Psi_{1}^{2}(\xi)\right\rangle=1,\left\langle\Psi_{2}^{2}(\xi)\right\rangle=2,\left\langle\Psi_{3}^{2}(\xi)\right\rangle=6, \ldots
$$

Suppose that we somehow know the values of the deterministic coefficients, $f_{i}$, for a given vector, $\mathbf{x}$. The statistics of the stochastic variable, $f^{*}(\mathbf{x}, \xi)$, such as mean and variance can be evaluated as follows.

$$
\begin{aligned}
\bar{f}^{*}=E\left(f^{*}(\xi)\right) & =\int_{-\infty}^{\infty} f^{*}(\xi) p_{N}(\xi) d \xi=f_{0} \\
\operatorname{Var}\left(f^{*}(\xi)\right) & =E\left(\left[f^{*}(\xi)-\bar{f}^{*}\right]^{2}\right) \\
& =\int_{-\infty}^{\infty}\left[f^{*}(\xi)-\bar{f}^{*}\right]^{2} p_{N}(\xi) d \xi \\
& =\sum_{i=1}^{P} f_{i}^{2}\left\langle\Psi_{i}^{2}(\xi)\right\rangle
\end{aligned}
$$

where we used the orthogonality of $\Psi_{i}$ and the fact that $E\left(\Psi_{i}\right)=0, i>0$. Since the inner product terms, $\left\langle\Psi_{i}^{2}\right\rangle$, are known a priori from Eq. 5 , the mean and the variance can be expressed as functions of deterministic coefficients $f_{i}$ where the leading coefficient $f_{0}$ represents the mean and those of the higher-order terms contributing to the variance.

For Eqs. 6 and 7 to be used for the evaluation of mean and variance, the deterministic coefficients must be known. This is where various methods based on Polynomial Chaos Expansion differ. The simplest approach is by Walters, et al. [5], in which $P+1$ samples are first chosen 


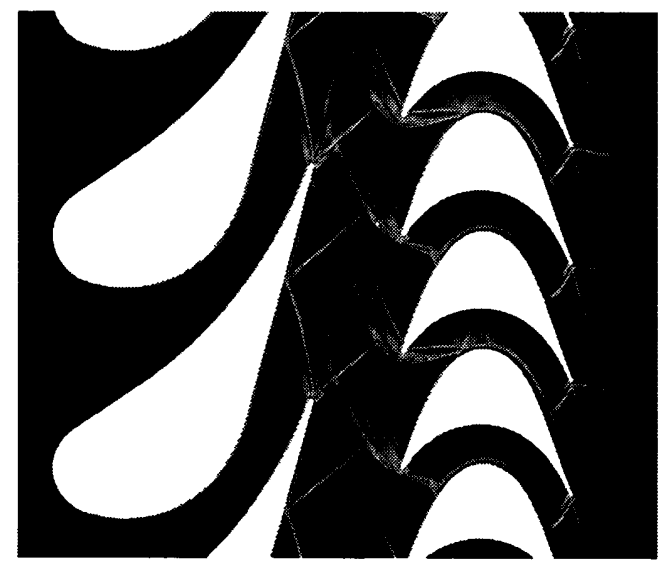

Figure 1: Blah
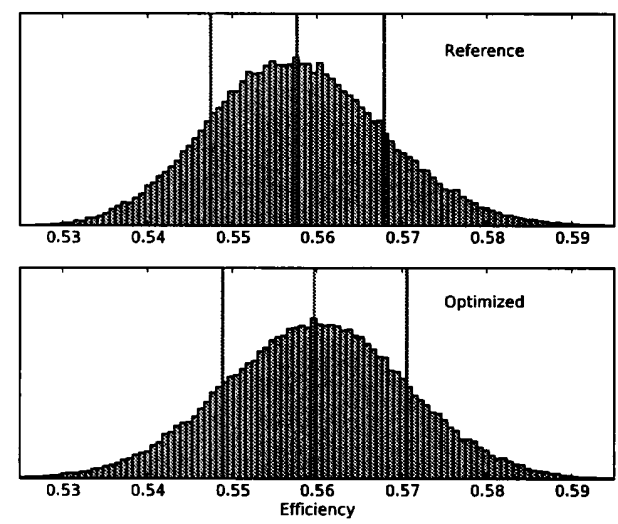

Figure 2: Blah for $\xi=\left\{\xi_{0}, \xi_{1}, \ldots, \xi_{p}\right\}$ and numerical simulation is carried out for each sample of $\xi$. This results in the following system of linear equations.

$\left[\begin{array}{cccc}\Psi_{0}\left(\xi_{0}\right) & \Psi_{1}\left(\xi_{0}\right) & \ldots & \Psi_{P}\left(\xi_{0}\right) \\ \Psi_{0}\left(\xi_{1}\right) & \Psi_{1}\left(\xi_{1}\right) & \ldots & \Psi_{P}\left(\xi_{1}\right) \\ \vdots & \vdots & \ddots & \vdots \\ \Psi_{0}\left(\xi_{p}\right) & \Psi_{1}\left(\xi_{p}\right) & \ldots & \Psi_{P}\left(\xi_{p}\right)\end{array}\right]\left\{\begin{array}{c}f_{0} \\ f_{1} \\ \vdots \\ f_{p}\end{array}\right\}=\left\{\begin{array}{c}f^{*}\left(\xi_{0}\right) \\ f^{*}\left(\xi_{1}\right) \\ \vdots \\ f^{*}\left(\xi_{p}\right)\end{array}\right\}$

where the right hand side vector represents the results of numerical simulation corresponding to each realization of random samples. The deterministic coefficients, $f_{i}$, are obtained by solving Eq. 8. Note that the solution of Eq. 8 depends on the sampling method used.

If more than $P+1$ samples are taken, we have an overdetermined system of equations for which least square approach can be used to solve for the best deterministic coefficient vector. As with the previous approach, the choice of sampling method is left open. Hosder et al. [6] investigated the influence of different sampling methods. The conference presentation will compare the results of the previous approach with the sampling-based approach, as well as with Monte-Carlo simulations.

\section{Numerical Results}

In order to assess uncertainty propagation by PCE, flow field inside the first stage of NASA M-1 fuel turbine was simulated by ANSYS CFX, a commercial CFD code. The turbine is a supersonic impulse turbine which produces complex shock-shock and shock-boundary layer interactions as seen in Fig. 1. Total-to-static efficiency was computed for this turbine under uncertainty in the rotational speed. Figure 2 shows a comparison of the mean and variance estimated by the PCE method for two turbine geometries. The conference presentation will also show the results of the over-sampling approach and compare them with Monte-Carlo simulations.

\section{Concluding Remarks}

The present study compared real-world performance of several PCE-based uncertainty propagation methods, as applied to a supersonic impulse turbine stage efficiency under uncertain operation conditions. Though computational advantage over Monte Carlo simulation is obvious, future research should focus also on the management of computational cost when more than one random variable is involved as is typically the case with any fluid machine design problem.

\section{Acknowledgement}

This study was partially subsidized by JKA through its promotion funds from KEIRIN RACE.

\section{References}

[1] Drela, M., "Pros and Cons of Airfoil Optimization," Frontiers of Computational Fluid Dynamics, edited by D. A. Caughey and M. M. Hafez, World Scientific, 1998, pp. 363-381.

[2] Wiener, N., "The Homogeneous Chaos," American Journal of Mathematics, Vol. 60, No. 4, 1938, pp. 897-936.

[3] Ghanem, R. G. and Spanos, P. D., Stochastic Finite Elements : A Spectral Approach, Springer-Verlag, 1991.

[4] Hosder, S., Walters, R. W., and Balch, M., "PointCollocation Nonintrusive Polynomial Chaos Method for Stochastic Computational Fluid Dynamics," AIAA Journal, Vol. 48, No. 12, 2010, pp. 2721-2730.

[5] Walters, R. W., "Optimization and Reconstruction via the Polynomial Chaos," Proceedings of the 6th European Conference on Structural Dynamics, Paris, France, Sept. 2005.

[6] Hosder, S., Walters, R. W., and Balch, M., "Efficient Sampling for Non-Intrusive Polynomial Chaos Application with Multiple Uncertain Input Variables," AIAA Paper 2007-1939, April 2007. 